\title{
Acute Liver Failure and Thyrotoxicosis Managed with Liver Transplant and Thyroidectomy
}

\author{
Gerardo Tamayo-Enriquez*, Lyle J Burdine, Emmanouil Giorgakis and Gary W Barone
}

Department of Surgery, USA

*Corresponding author: Gerardo Tamayo-Enriquez, Department of Surgery, USA

\begin{abstract}
Acute liver failure and hyperthyroidism are not typically common, although some cases have been reported. The mechanisms involved and optimal management are not well-defined. This article presents the case of a 32-year-old African American female referred for evaluation of abdominal pain and jaundice, with a past medical history of systemic lupus erythematosus and Grave's disease. She had thyrotoxicosis after administration of contrast and developed acute liver failure culminating in liver transplant and subsequent total thyroidectomy with a favorable outcome.
\end{abstract}

Keywords: Grave's Disease; Autoimmune Hepatitis; Thyrotoxicosis; Acute Liver Failure; Liver Transplant; Thyroidectomy; Contrast Induced

Abbreviations: OLT: Orthotopic liver transplant; SLE: Systemic Lupus Erythematosus; TT: Total thyroidectomy; HD: Hospital day; POD: Post-operative day; ANA: Anti-nuclear antibodies; NaMELD: Sodium model for end-stage liver disease; PTU: Propylthiouracil.

\section{Introduction}

The association between liver function abnormalities and thyroid dysfunction is well established dating back to at least 1874 when Habershon presented a case of exophthalmic goiter and jaundice to the London Medical Society [1]. The range of liver dysfunction can go from mild elevation of hepatic enzymes to acute liver failure, which is defined as the presence of hepatic encephalopathy within 8 weeks of initial symptoms in a patient without underlying liver disease. Multiple scales have been devised to predict mortality and determine which patients would benefit from orthotopic liver transplant (OLT), with the presence of hepatic encephalopathy as a key indicator [2]. Here we present a case of a 32-year-old female with systemic lupus erythematosus (SLE) and exacerbation of Grave's disease after iodinated contrast that presented with acute liver failure and thyrotoxicosis requiring OLT and total thyroidectomy (TT) after medical management of both conditions.

Case

A 32-year-old African American female was referred to the emergency room for evaluation of a 3-week history of worsening abdominal pain and jaundice. She also reported loss of appetite and occasional epigastric discomfort. Her past medical history included SLE and Grave's disease [3]. Both conditions were well controlled, and her medications included multivitamins, hydroxychloroquine and ferrous sulfate. Three weeks prior to admission she underwent a CT scan with iodinated contrast for abdominal pain at an outside hospital. At that time, she received prednisone (60mg PO qd) and diphenhydramine for a presumed diagnosis of autoimmune hepatitis with positive antinuclear antibodies (ANA) at 1:320. Ferritin, alpha 1 antitrypsin, acetaminophen and viral serologies were all negative prior to admission. Her initial vital signs showed BP 110/70 mmHg, HR 134 bpm, RR 20 bpm, T 37 ${ }^{\circ} \mathrm{C}$ and SpO2 100\%. Physical examination was significant for generalized jaundice and moderate abdominal pain on palpation.

At that time her laboratories were INR 3.8, WBC $36.6 \mathrm{k} / \mathrm{uL}, \mathrm{K}$ $2.7 \mathrm{mmol} / \mathrm{L}$, lactate $2.4 \mathrm{mmol} / \mathrm{L}$, Alk Phos $187 \mathrm{IU} / \mathrm{L}$, AST $625 \mathrm{IU} / \mathrm{L}$, $872 \mathrm{IU} / \mathrm{L}$, Built $30.2 \mathrm{mg} / \mathrm{dL}$, Bild $21.9 \mathrm{mg} / \mathrm{dL}$, T3 $11.3 \mathrm{pg} / \mathrm{mL}$, T4 $4.46 \mathrm{ng} / \mathrm{dL}$, TSH $0.02 \mathrm{uIU} / \mathrm{mL}$ and ceruloplasmin $26 \mathrm{mg} / \mathrm{dL}$. On HD \#11 her 24hr copper excretion was $241.6 \mathrm{ug} / \mathrm{d}$. Her admission Named was 35 and 31 at time of transplant. Renal function 
remained normal throughout her hospitalization. Trends for her hospital stay are shown in (Tables 1 \& 2) After initial stabilization, Doppler ultrasound revealed patent vasculature, and MRI showed nodular appearance of the liver consistent with edema or early cirrhosis (Figure 1). The liver transplant service was consulted and a standard workup for potential liver transplant was started.

Table 1: Liver Function Tests. OLT: Orthotopic Liver Transplant. TT: Total Thyroidectomy.

\section{Liver Function Tests}

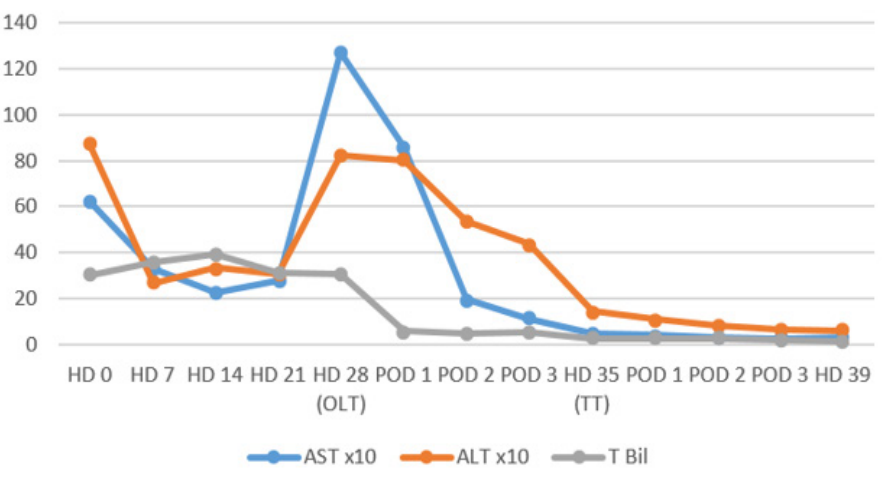

Table 2: TSH remained undetectable at $<0.02$.

Thyroid Function/INR
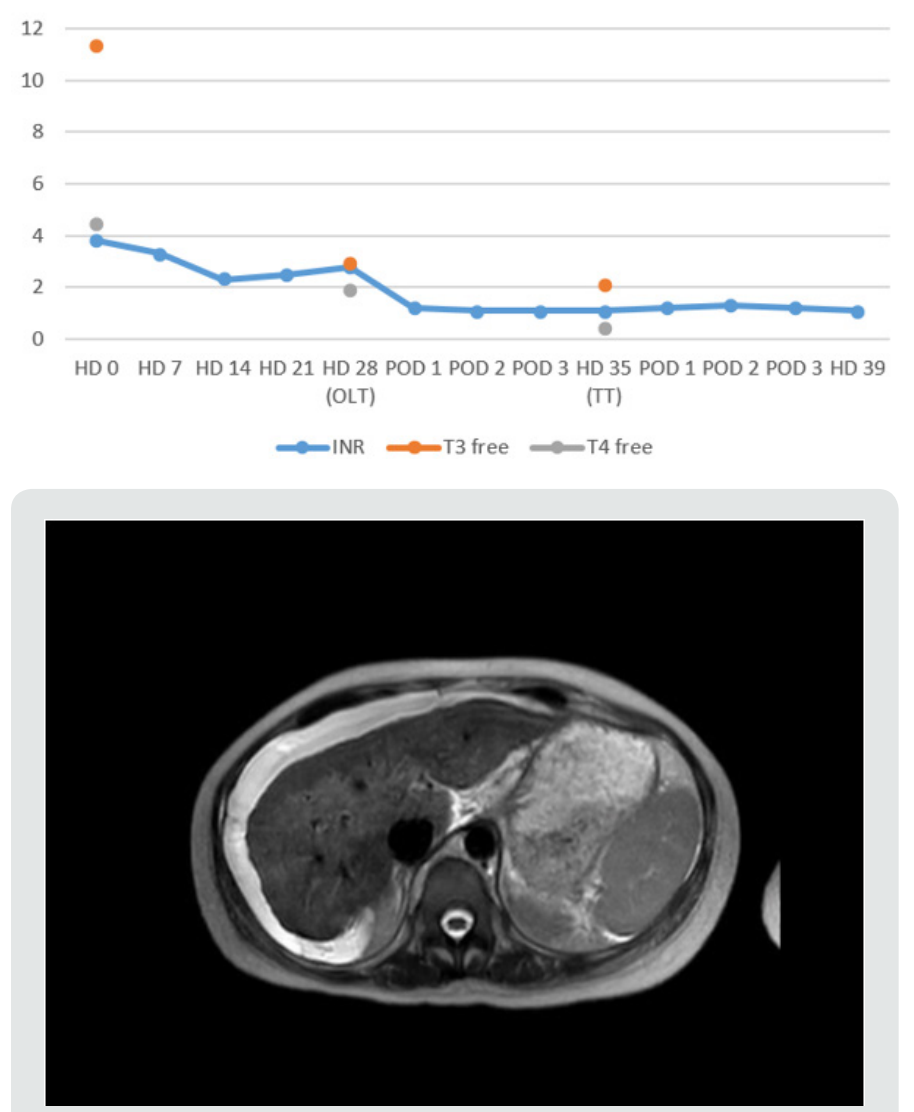

Figure 1: MRI of abdomen showing nodular appearance of liver consistent with edema or early cirrhosis.

A transvenous liver biopsy on HD \#6 was positive for marked active hepatitis with bridging necrosis, parenchymal collapse and minimal focal staining for copper. At the time of biopsy, a hemodynamic study was performed consistent with portal hypertension (Figure 2). Blood cultures were positive for E. coli and urine cultures for E. faecalis on admission, and she received ceftriaxone and meropenem with negative conversion of cultures. Her condition continued to deteriorate with worsening hepatic encephalopathy despite optimal treatment with rifaximin and lactulose. Oral prednisone was continued at $40 \mathrm{mg}$ PO/qd. Her thyrotoxicosis was managed with propranolol and potassium iodide oral solution. An echocardiogram ruled out congestive heart failure. At HD \#26 she was admitted to the intensive care unit and due to her condition, it was deemed she was at risk of death within one week and was listed as emergency status $1 \mathrm{~A}$ on the united network for organ sharing.

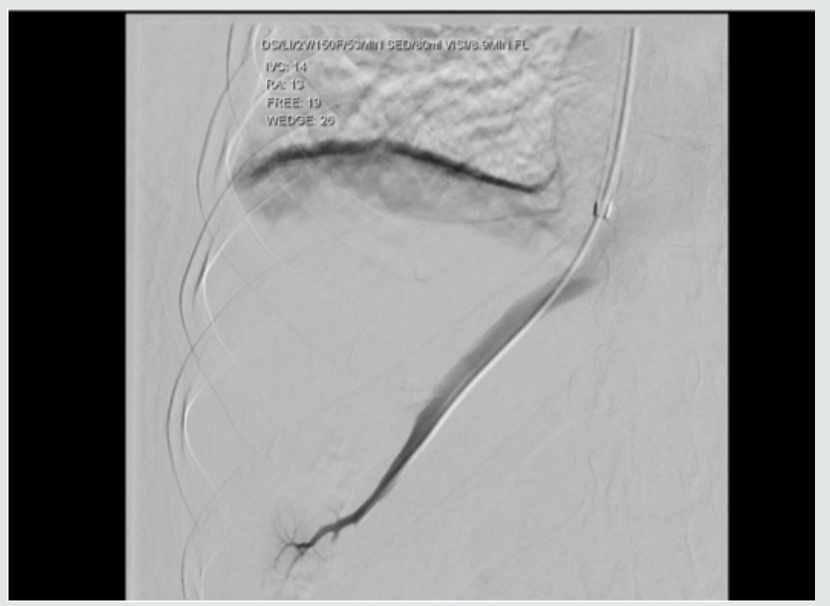

Figure 2: Transvenous hemodynamic study consistent with portal hypertension. Free hepatic pressure $19 \mathrm{mmHg}$, wedge hepatic pressure $26 \mathrm{mmHg}$, hepatic venous gradient $7 \mathrm{mmHg}$.

A suitable donor became available on HD \#28 and she underwent OLT with methylprednisolone and mycophenolate induction without complications. She received $250 \mathrm{mcg}$ of potassium iodide the night prior to transplant. Liver explant revealed extensive bridging necrosis, parenchymal collapse, no definite areas of fibrosis and minimal copper staining. She recovered without incidents from transplant and underwent total thyroidectomy on POD \#7, she was then started on levothyroxine. She was discharged on HD \#39 to an acute rehabilitation facility. At 1-year follow up, she has excellent graft function, remains euthyroid and has not experienced flare ups of SLE.

\section{Discussion}

Exposure to iodinated contrast is well known to cause thyroid dysfunction, with an iodine content of 320 to $370 \mathrm{mg} / \mathrm{mL}$ it is well above the recommended daily allowance and can precipitate thyrotoxicosis via the Jöb-Basedow phenomenon in patients with Grave's disease [3]. Hyperthyroidism, particularly in Grave's disease, is associated with primary biliary cirrhosis and autoimmune hepatitis. In patients without heart failure and hyperthyroidism, the liver biopsy has demonstrated fatty infiltration, cytoplasmic vacuolization, nuclear irregularity and hyperchromatism [4]. 
Although the exact mechanism for the observed liver-thyroid interactions is unknown, the following have been proposed:

a. systemic effects of thyroid excess,

b. direct toxic effects of thyroid hormone,

c. intrinsic liver and thyroid autoimmune mechanisms,

d. abnormal thyroid metabolism due to liver disease, and

e. subclinical physiologic effects of thyroid hormone [5].

A feasible explanation is the presence of a hypermetabolic state with increased hepatic oxygen consumption, but without increases in hepatic blood flow, affecting the centrilobular zones and interfering with bile transport. These findings are consistent with the picture of cholestasis usually present in such cases [6]. In a series of 84 patients with acute liver failure, Anastasiou and colleagues reported a 50\% incidence of thyroid hormone abnormalities, with a worse outcome in patients with hyperthyroidism, believed to be secondary to an increase in oxygen consumption and decreased organ perfusion [7]. In the current case, the presence of drug-induced liver injury had to be ruled out, hydroxychloroquine has been associated with acute liver failure requiring liver transplantation, but it appears within two weeks of starting therapy [8]. There is a well-known correlation between anti-thyroid medications and hepatic dysfunction, for example, propylthiouracil (PTU) and methimazole are associated with an incidence of severe liver injury in $0.1 \%$ and $0.1-0.2 \%$ of patients, respectively [9].

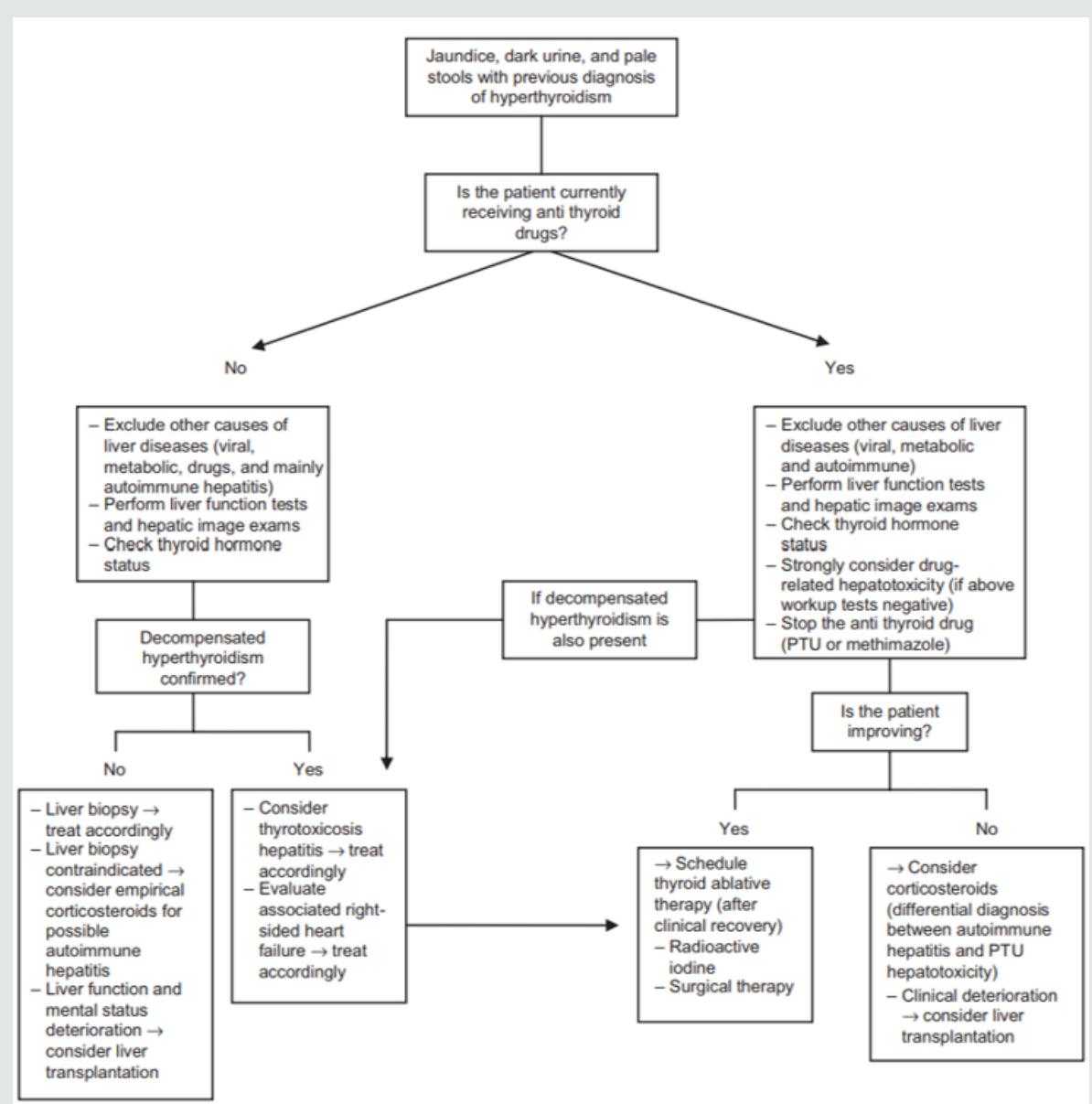

Figure 3: Proposed algorithm for management of patients with symptoms of liver dysfunction and previous diagnosis of hyperthyroidism. [Figure note: Figure obtained from de Campos Mazo, 2013; @ 2013 Mazo et al, publisher and licensee Dove Medical Press Ltd. This is an Open Access article which permits unrestricted noncommercial use, provided the original work is properly cited.] [10].

To our knowledge, our patient was not exposed to these medications. In the absence of established guidelines, a useful algorithm for the management of liver dysfunction with hyperthyroidism has been proposed, (Figure 3) This algorithm is based on a case series of 8 patients in which two cases developed acute liver failure suitable for OLT [10]. Nonetheless, the timing and sequence of OLT and thyroidectomy is not well established. Although it has been proposed not to delay the thyroidectomy as it might represent a potentially life-saving procedure [11]. The incidence of acute liver failure in the setting of hyperthyroidism remains a rare event, with patients undergoing OLT and thyroidectomy even more unusual. In this case report, adequate control of the thyrotoxicosis was achieved prior to transplant with oral potassium iodide and propranolol. In this patient with rapidly progressive liver failure, 
it appeared clinically prudent to undergo emergent OLT followed by TT.

\section{Conclusion}

The presence of ANA in a patient with SLE and Grave's disease suggest the diagnosis of autoimmune hepatitis, however in this case report, the administration of iodinated contrast and the presence of abnormal copper metabolism made the diagnosis more difficult. The optimal management of these patients is not well established, with only a few cases reported in the literature. Based on our experience, medical stabilization followed by OLT and TT appears to have been an adequate course of treatment. Further studies are needed to help determine guidelines.

\section{References}

1. Habershon SO (1874) Exophtalmic goiter heart disease jaundice. death Lancet 1: 510-512.

2. Bernal W, Wendon J (2013) Acute liver failure. N Engl J Med 369(26): 2525-2534.

3. Lee SY, Rhee CM, Leung AM, Braverman LE, Brent GA, et al. (2015) A review: Radiographic iodinated contrast media-induced thyroid dysfunction. J Clin Endocrinol Metab 100(2): 376-383.
4. Khemichian S, Fong TL (2011) Hepatic dysfunction in hyperthyroidism. Gastroenterol Hepatol 7(5): 337-339.

5. Choudhary AM, Roberts I (1999) Thyroid storm presenting with liver failure. J Clin Gastroenterol 29(4): 318-321.

6. Bal CS, Chawla M (2010) Hyperthyroidism and jaundice. Indian J Nucl Med 25(4): 131-134.

7. Anastasiou O, Sydor S, Sowa JP, Paul Manka, Antonios Katsounas, et al. (2015). Higher thyroid-stimulating hormone, triiodothyronine and thyroxine values are associated with better outcome in acute liver failure. PLoS ONE 10(7).

8. Makin AJ, Wendon J, Fitt S, Portmann BC, Williams R (1994) Fulminant hepatic failure secondary to hydroxychloroquine. Gut 35(4): 569-570.

9. Heidari R, Niknahad H, Jamshidzadeh A, Abdoli N (2014) Factors affecting drug-induced liver injury: antithyroid drugs as instances. Clin Mol Hepatol 20(3): 237-248.

10. de Campos Mazo DF, de Vasconcelos GB, Pereira MA, de Mello ES, Bacchella T, et al. (2013) Clinical spectrum and therapeutic approach to hepatocellular injury in patients with hyperthyroidism. Clin Exp Gastroenterol 6: 9-17.

11. Kandil E, Khalek MA, Thethi T, Abd Elmageed Z, Khan A, et al. (2011) Thyroid storm in a patient with fulminant hepatic failure. Laryngoscope 121(1): 164-166.

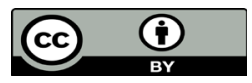

This work is licensed under Creative Commons Attribution 4.0 License

To Submit Your Article Click Here:

Submit Article

DOI: $10.32474 /$ SCSOAJ.2019.02.000127

$\begin{gathered}\text { Surgery \& Case Studies: Open } \\ \text { Access Journal }\end{gathered}$
Assets of Publishing with us
- Global archiving of articles
- Immediate, unrestricted online access
- Rigorous Peer Review Process

